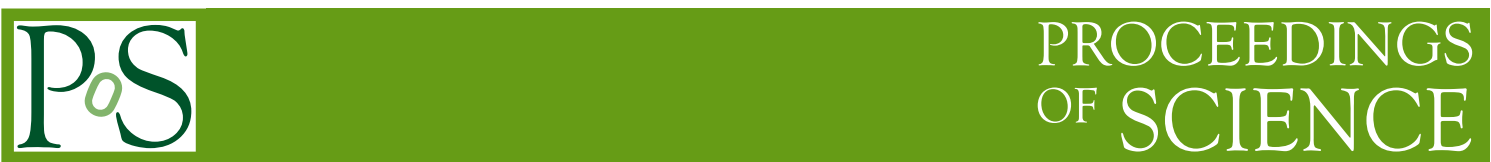

\title{
Measurement Of The Proton Spin Polarisabilities
}

\section{D.G. Middleton*}

Institut für Kernphysik, Universität Mainz/Mt. Allison University

E-mail: middletn@kph.uni-mainz.de

\section{P.P. Martel}

University of Massachusetts, Amherst

on behalf of the A2 collaboration.

\begin{abstract}
The double polarisation asymmetry, $\Sigma_{2 x}$, for the Compton scattering reaction off the proton has been measured for the first time for an incident photon energy range of $273 \leq E_{\gamma} \leq 303 \mathrm{MeV}$ and a scattered photon angular range of $80 \leq \theta_{\gamma^{\prime}} \leq 160^{\circ}$. This has allowed one of the four proton spin polarisabilities, $\gamma_{E 1 E 1}$, to be extracted individually for the first time. Comparison to dispersion relation calculations indicate that a value of $\gamma_{E 1 E 1}=-4.3 \pm 1.5 \times 10^{-4} \mathrm{fm}^{4}$ best describes the measured asymmetries.
\end{abstract}

51st International Winter Meeting on Nuclear Physics

21-25 January 2013

Bormio (Italy)

\footnotetext{
* Speaker.
} 


\section{Introduction}

Nucleon polarisabilities are fundamental structure observables related to the nucleon's internal dynamics and describe its response to an applied electric or magnetic field. These polarisabilities can be measured by elastically scattering real photons off of the nucleon in what is known as the Compton scattering reaction. The amplitude of the Compton scattering reaction from the nucleon can be expanded in terms of the incident photon energy, $E_{\gamma}$. The zeroth order term is the typical Thompson scattering term for scattering of a point-like particle of given charge and mass. Using the notation of Levchuck and L'vov [1] the zeroth order Hamiltonian has the form

$$
H_{\mathrm{eff}}^{(0)}=\frac{\vec{\pi}^{2}}{2 m}+e \phi
$$

where $m$ is the mass and $e$ the electric charge and

$$
\vec{\pi}=\vec{p}-e \vec{A}
$$

is the covariant momentum where $\vec{p}$ is the momentum and $\vec{A}$ is the vector potential. With expansion to first order the Hamiltonian has the form

$$
H_{\mathrm{eff}}^{(1)}=-\frac{e(1+\kappa)}{2 m} \vec{\sigma} \cdot \vec{H}-\frac{e(1+2 \kappa)}{8 m^{2}} \vec{\sigma} \cdot[\vec{E} \times \vec{\pi}-\vec{\pi} \times \vec{E}]
$$

where $\kappa$ is the anomalous magnetic moment of the nucleon. The zeroth and first order, or so-called Born terms, represent the external degrees of freedom of the nucleon and are the only effective terms for $E_{\gamma} \leq 20 \mathrm{MeV}$ [2]. At higher $E_{\gamma}$ internal degrees of freedom start to play a role. This is reflected in the second order term of the expansion

$$
H_{\mathrm{eff}}^{(2)}=-4 \pi\left[\frac{1}{2} \alpha_{E 1} \vec{E}^{2}+\frac{1}{2} \beta_{M 1} \vec{H}^{2}\right]
$$

which contains $\alpha_{E 1}$ and $\beta_{M 1}$ which are the electric and magnetic polarisabilities, collectively known as the scalar polarisabilities. These describe the nucleon's internal response to an applied electromagnetic field [3]. The proton scalar polarisabilities have been measured many times since the 1950s using Compton scattering off an unpolarised proton target; a recent review of these experiments can be found in [4]. The current Particle Data Group values for $\alpha_{E 1}$ and $\beta_{M 1}$ are

$$
\begin{aligned}
& \alpha_{E 1}=[12.1 \pm 0.3(\text { stat }) \mp 0.4(\text { syst }) \pm 0.3(\mathrm{mod})] \times 10^{-4} \mathrm{fm}^{3} \\
& \beta_{M 1}=[1.6 \pm 0.4(\text { stat }) \pm 0.4(\text { syst }) \pm 0.4(\mathrm{mod})] \times 10^{-4} \mathrm{fm}^{3}
\end{aligned}
$$

and are based on the results of a measurement by Olmos de León et al. [5] where in their final analysis they also included results from three earlier measurements $[6,7,8]$.

When the Compton scattering amplitude is expanded to third order the spin (or vector) polarisabilities (SPs) come into play 


$$
\begin{aligned}
H_{\mathrm{eff}}^{(3)}=-4 \pi[ & \frac{1}{2} \gamma_{E 1 E 1} \vec{\sigma} \cdot(\vec{E} \times \dot{\vec{E}})+\frac{1}{2} \gamma_{M 1 M 1} \vec{\sigma} \cdot(\vec{H} \times \dot{\vec{H}}) \\
& \left.-\gamma_{M 1 E 2} E_{i j} \sigma_{i} H_{j}+\gamma_{E 1 M 2} H_{i j} \sigma_{i} E_{j}\right]
\end{aligned}
$$

which are $\gamma_{E 1 E 1}, \gamma_{M 1 M 1}, \gamma_{M 1 E 2}$ and $\gamma_{E 1 M 2}$ in the above equation. These describe the nucleon's spin response to an applied electric or magnetic field [3]. These have not been individually measured for the proton though two linear combinations of them have been: the forward and backward SPs, $\gamma_{0}$ and $\gamma_{\pi}$ respectively. These combinations of the SPs and the current values for them are

$$
\begin{gathered}
\gamma_{0}=-\gamma_{E 1 E 1}-\gamma_{E 1 M 2}-\gamma_{M 1 E 2}-\gamma_{M 1 M 1}=(-1.0 \pm 0.08) \times 10^{-4} \mathrm{fm}^{4} \\
\gamma_{\pi}=-\gamma_{E 1 E 1}-\gamma_{E 1 M 2}+\gamma_{M 1 E 2}+\gamma_{M 1 M 1}=(8.0 \pm 1.8) \times 10^{-4} \mathrm{fm}^{4}
\end{gathered}
$$

$\gamma_{0}$ was determined from data taken by the GDH collaboration $[9,10]$ to study the Gerasimov-DrellHearn sum rule while $\gamma_{\pi}$ was determined with a dispersive analysis of backward-angle Compton scattering data taken at MAMI [11]. It should be noted that this value of $\gamma_{\pi}$ does not include the $\pi^{0}$-pole term where $\gamma_{\pi}^{\pi^{0}-\text { pole }}-46.7 \times 10^{-4} \mathrm{fm}^{4}$ [12].

There are various theoretical predictions for the different scalar and spin polarisabilities and a review of some of these can be found in [4]. The two main approaches used to study nucleon polarisabilities are based on Chiral Perturbation Theory $(\chi \mathrm{PT})$ and dispersion relations; a summary of the different predicted values can be seen in table 1 taken from [13]. As can be seen there are a wide range of predicted values for the different SPs so experimental determination of them individually would help improve theoretical approaches to this reaction.

To individually determine each of the four SPs for the proton three separate Compton scattering asymmetry measurements can be used, labelled $\Sigma_{2 x}, \Sigma_{2 y}$ and $\Sigma_{3} . \Sigma_{2 x}$ measures the asymmetry in the response of circularly polarised photons incident upon a transversely polarised target. $\Sigma_{2 y}$ is similar to $\Sigma_{2 x}$ except that the target is longitudinally polarised. $\Sigma_{3}$ is a measurement of the beam asymmetry and uses linearly polarised photons incident upon an unpolarised target. The rest of this

\begin{tabular}{|c|c|c|c|c|c|c|c|c|c|}
\hline & $\mathrm{O}\left(\mathrm{p}^{3}\right)$ & $\mathrm{O}\left(\mathrm{p}^{4}\right)(1)$ & $\mathrm{O}\left(\mathrm{p}^{4}\right)(2)$ & LC3 & LC4 & SSE & BGLMN & HDPV & KS \\
\hline$\gamma_{E 1 E 1}$ & -5.7 & -1.4 & -1.8 & -3.2 & -2.8 & -5.7 & -3.4 & -4.3 & -5.0 \\
\hline$\gamma_{E 1 M 2}$ & 1.1 & 0.2 & 0.7 & 0.7 & 0.8 & .98 & 0.3 & -0.01 & -1.8 \\
\hline$\gamma_{M 1 E 2}$ & 1.1 & 1.8 & 1.8 & 0.7 & 0.3 & .98 & 1.9 & 2.1 & 1.1 \\
\hline$\gamma_{M 1 M 1}$ & -1.1 & 3.3 & 2.9 & -1.4 & -3.1 & 3.1 & 2.7 & 2.9 & 3.4 \\
\hline$\gamma_{0}$ & 4.6 & -3.9 & -3.6 & 3.1 & 4.8 & .64 & -1.5 & -0.7 & 2.3 \\
\hline$\gamma_{\pi}$ & 4.6 & 6.3 & 5.8 & 1.8 & -0.8 & 8.8 & 7.7 & 9.3 & 11.3 \\
\hline
\end{tabular}

Table 1: Theoretical values for the spin polarisabilities. $\mathrm{O}\left(\mathrm{p}^{3}\right), \mathrm{O}\left(\mathrm{p}^{4}\right)(1)$ and $\mathrm{O}\left(\mathrm{p}^{4}\right)(2)$ are $\chi \mathrm{PT}$ calculations from [14], [15] and [16] respectively. LC3 and LC4 are $\mathrm{O}\left(\mathrm{p}^{3}\right)$ and $\mathrm{O}\left(\mathrm{p}^{4}\right)$ Lorentz invariant $\chi \mathrm{PT}$ calculations, respectively [17]. SSE is a Small Scale Expansion calculation [14]. The remaining three are all dispersion relation calculations [18][19][20][21]. 
article describes the current state of the analysis from a first measurement of $\Sigma_{2 x}$ and is based on the work of P.P. Martel [13].

\section{Experimental Set-Up}

The measurement was carried out at the real photon facility of the A2 collaboration at the Mainzer Mikrotron (MAMI) [22]. A beam of circularly polarised Bremsstrahlung photons was produced by directing a beam of longitudinally polarised electrons of energy $450 \mathrm{MeV}$ against a $10 \mu \mathrm{m}$ copper radiator. The photon energy was determined by momentum analysing the recoil electrons using the Glasgow-Mainz Tagged Photon Spectrometer [23, 24, 25] and photon energy resolutions of $0.5-1.5 \mathrm{MeV}$ were achieved. The photon beam was collimated and directed to the experimental area. The fraction of tagged photons passing through the collimator was measured at a reduced beam current by moving a $\sim 100 \%$ efficient lead glass detector into the photon beam. An ionisation chamber monitored the photon flux during the experiment. The electron beam polarisation was measured periodically using a Mott polarimeter located in the electron beam line [26].

The reaction particles were detected using the Crystal Ball (CB) [27] and TAPS [28] detector set-up in the A2 hall which is shown schematically in figure 1. The CB comprises a segmented calorimeter of $672 \mathrm{NaI}$ crystals covering an angular range of $21^{\circ} \leq \theta \leq 159^{\circ}$ which covers $94 \%$ of $4 \pi$ steradians. Each NaI crystal has separate TDC and ADC readouts giving a time resolution of $2 \mathrm{~ns}$, an angular resolution of $\Delta \theta \approx 2.5^{\circ}$ and energy resolution of $\Delta E \approx 3 \%$. A Particle Identification Detector (PID) [29], consisting of 24 EJ204 plastic scintillators arranged in a cylinder which surrounded the target, was used to identify charged particles. The type of particle was determined using the differential energy loss measured in the PID detector, together with the energy deposited in the CB. Around the PID were a set of two coaxial cylindrical MultiWire Proportional Chambers (MWPC) for charged particle tracking of similar design to those described in [30]. Each of the MWPCs consist of three layers: inner and outer cathode strips and an anode wire layer. The wires of the anode layer are stretched parallel to the cylinder axis while the cathode strips are wound helically at an angle of $\pm 45^{\circ}$ with respect to the anode wires. The MWPC has a resolution of $\Delta \theta \approx 2^{\circ}$ and $\Delta \phi \approx 3^{\circ}$. Downstream of the CB was the TAPS detector array which covered an angular range of $\theta \leq 20^{\circ}$ except for a small hole around the photon beam line. TAPS consists of 366 hexagonal $\mathrm{BaF}_{2}$ crystals and two inner rings of $72 \mathrm{PbWO}_{4}$ crystals. Each $\mathrm{BaF}_{2}$ crystal and set of $4 \mathrm{PbWO}_{4}$ crystals has its own $5 \mathrm{~mm}$ thick veto paddle for charged particle identification. TAPS has an angular resolution of $\Delta \theta \approx 0.7^{\circ}$ and energy resolution of $\Delta E \approx 3 \%$. In addition to the above detectors a Čerenkov detector [13] was positioned in the downstream hole of the CB in front of TAPS. This was was used to veto electrons originating from pair production in the target.

For the majority of the data taking a butanol 'frozen-spin target' (FST) [31] was used to provide the polarised hydrogen necessary for the measurement. This was operated at $0.025 \mathrm{~K}$ and consisted of beads of polarised butanol in a liquid ${ }^{3} \mathrm{He} /{ }^{4} \mathrm{He}$ bath. The FST target cell was $2 \mathrm{~cm}$ in length and had a target thickness of $N_{T} \approx 9.1 \times 10^{22} \mathrm{~cm}^{-2}$ with an average proton polarisation of $P_{p}=70 \%$. To polarise the hydrogen of the butanol the FST was first cooled to $0.2 \mathrm{~K}$ and then polarised using the 'Dynamic Nuclear Polarisation' process in a magnetic field of $2.5 \mathrm{~T}$ using $70 \mathrm{GHz}$ microwaves. During the measurement the target was further cooled to $0.025 \mathrm{~K}$ and placed in a holding field of 


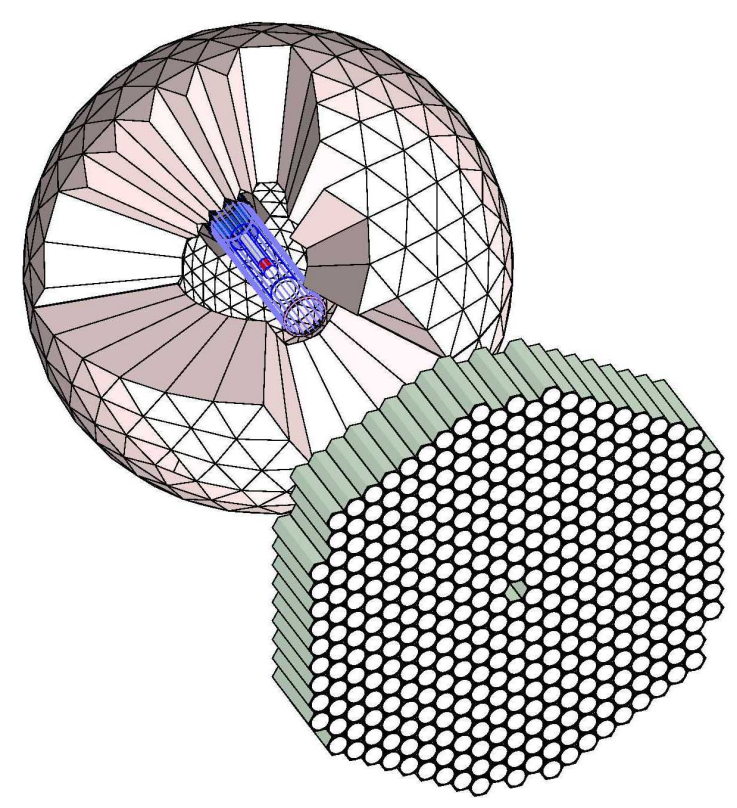

Figure 1: A schematic diagram of the Crystal Ball-TAPS set-up in A2 with the beam travelling from top-left to bottom right. The PID and MWPCs can be seen in the centre of the CB. See the main text for more details.

$0.45 \mathrm{~T}$ giving it a relaxation time of around 1500 hours. Typically the target was repolarised every 5-6 days and the direction of the polarisation flipped during repolarisation to reduce systematic effects.

To account for reactions in the ${ }^{12} \mathrm{C}$ and ${ }^{16} \mathrm{O}$ in the FST, data were taken with a 'carbon-foam' target in place within the FST cryostat. The length was the same as the FST and the density chosen so that the number of nuclei would match the total number of non-hydrogen nuclei in the FST; see [13] for more details.

The trigger for the measurement was a simple CB energy sum which included all crystals in the $\mathrm{CB}$; the threshold was set at $E_{\text {sum }} \geq 100 \mathrm{MeV}$.

\section{Analysis}

To identify events of interest both of the final state particles from the Compton reaction had to be detected due to other competing reactions. The main one of these was the $\gamma p \rightarrow \pi^{0} p$ reaction, which has a cross section roughly one hundred times larger than the Compton one in the $E_{\gamma}$ region of interest. There were also events from heavier nuclei in the butanol that could contribute to background; data taken with the carbon-foam target were analysed in exactly the same way as those for the FST and subtracted to account for this. The carbon data were scaled to take into account the different luminosities for the two target settings. Full details of the data analysis can be found in the thesis of P.P. Martel [13]. 


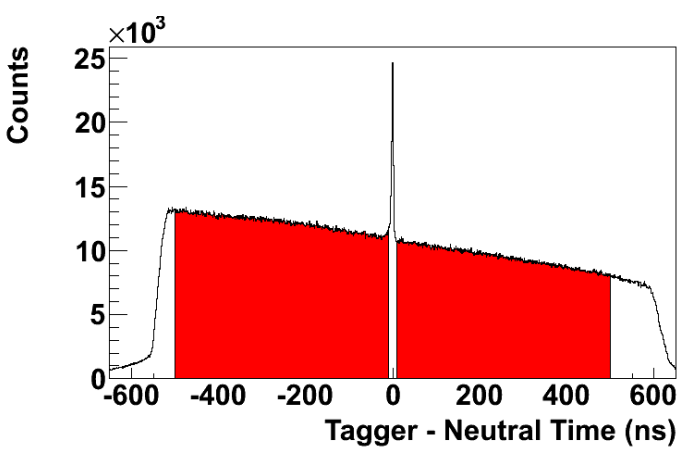

(a) Full $t_{e^{\prime}-\gamma^{\prime}}$

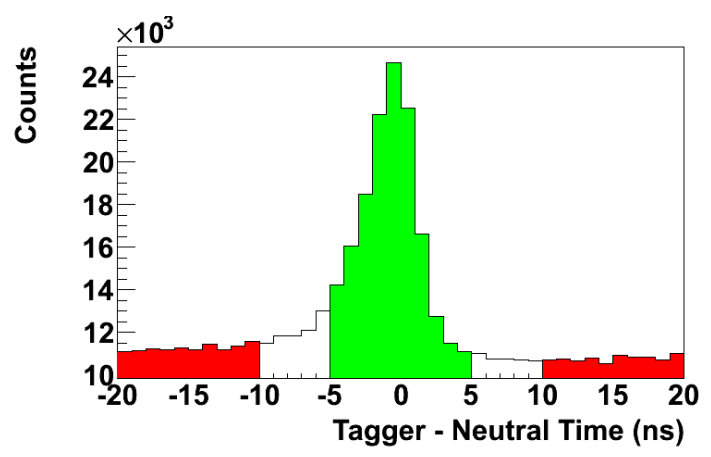

(b) Prompt $t_{e^{\prime}-\gamma^{\prime}}$

Figure 2: Coincidence time plots for an electron in the tagger and a photon in the CB. Figure (a) shows the full coincidence time range with the accidental sample region shaded red. Figure (b) shows an enlarged view of the prompt region with the prompt sample shaded green.

Candidate Compton events required there to be both a neutral and charged particle detected in coincidence within the CB-TAPS detector systems. A charged particle was identified by requiring a signal in either the PID and TAPS-veto detectors. The type of charged particle was determined using the energy deposited in said detector and its corresponding calorimetric detector. Apart from this requirement an opening angle cut was applied to the proton of $\pm 10^{\circ}$ from the expected trajectory to further help reject non-Compton events. As the analysis stands events with only one neutral and one charged particle were accepted for further analysis due to the high level of background; events with higher multiplicity are ignored in what is shown here.

For the events of interest the photon-electron coincidence time, $t_{e^{\prime}-\gamma^{\prime}}$, was recreated to correct for accidental coincidences; this is shown in figure 2. The full time range is shown in figure 2a and an enlarged view of the prompt peak region is shown in figure $2 \mathrm{~b}$. As can be seen there is a clear prompt peak with a FWHM of $\sim 5$ ns that sits atop events from accidental coincidences. The asymmetry in the peak arises from an improper treatment of multiple hits in the tagger; such events will be analysed properly in the final analysis. The prompt and accidental samples used for the correction are shown by the green and red shaded regions in the figures respectively. The accidental events were scaled to that of the prompt region and then subtracted from this.

To further identify events of interest the missing mass was determined on an event-by-event basis as defined by

$$
M_{\text {miss }}=\sqrt{\left(E_{\gamma}+M_{p}-E_{\gamma^{\prime}}\right)^{2}-\left(\vec{p}_{\gamma}-\vec{p}_{\gamma^{\prime}}\right)^{2}} \underset{\text { Compton }}{=} M_{p}
$$

where $E_{\gamma}$ and $\vec{p}_{\gamma}$ are the energy and momentum of the incident photon, $E_{\gamma^{\prime}}$ and $\vec{p}_{\gamma^{\prime}}$ are the energy and momentum of the scattered photon and $M_{p}$ is the proton mass. Only energy information from the scattered and incident photon and target mass were used in determining the missing mass as seen in equation 3.1. Although energy information for the scattered proton was determined including corrections for energy loss it was found that using this did not significantly help in the analysis [13]. The only constraint on the proton was that it was within the opening angle cut described above. 


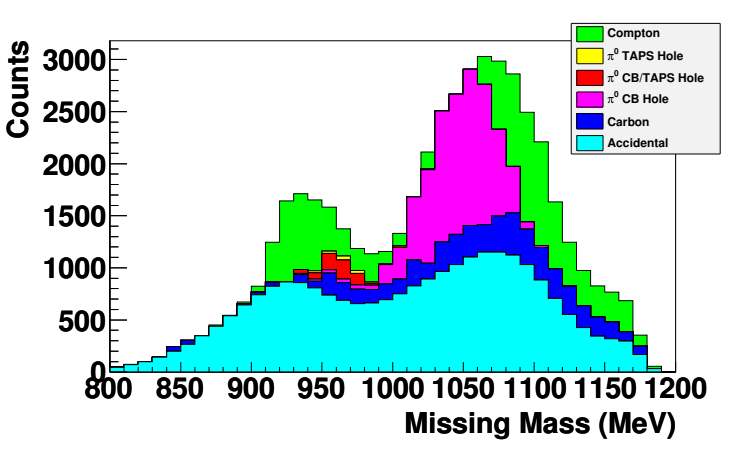

(a)

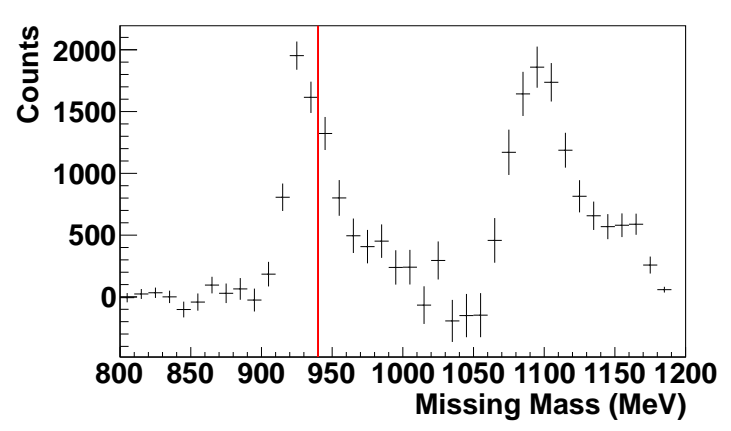

(b)

Figure 3: Missing mass for Compton events. Figure (a) shows the contribution of different types of events, see text for more details. Figure (b) shows the subtracted missing mass with statistical errors. The red line shows the cut applied for determination of the final asymmetries.

Figure 3 shows the missing mass distribution for $273 \leq E_{\gamma} \leq 303 \mathrm{MeV}$ and $100 \leq \theta_{\gamma^{\prime}} \leq 120^{\circ}$ where $\theta_{\gamma^{\prime}}$ is the scattered photon polar angle. Figure 3a shows the contribution of different prompt, accidental and background reaction events while figure $3 \mathrm{~b}$ shows the background subtracted missing mass. There are two distinct peaks in the missing mass spectrum of figure $3 \mathrm{a}$ at $\sim 940 \mathrm{MeV} / \mathrm{c}^{2}$ and $\sim 1070 \mathrm{MeV} / c^{2}$. The first peak is at the proton mass and is mainly from real Compton events while the higher energy peak is mainly from $\pi^{0}$-production events where one of the decay photons from the $\pi^{0}$ was lost to one of the three dead regions in the CB-TAPS set-up.

In figure $3 \mathrm{a}$ events from the accidental coincidence time regions are shown by the cyan shaded area and cover the full missing mass range. Events from heavier nuclei, as determined with the carbon-foam target, are shown by the blue shaded region. The yellow, red and magenta shaded regions are $\pi^{0}$-production events with a missing decay photon and represent the hole in TAPS around the photon beamline, the ring between the CB and TAPS and the upstream hole in the CB respectively. As can be seen the downstream dead regions mainly affect the lower energy peak and have a relatively small contribution. The events from upstream hole in the CB contribute the majority of strength of the peak centred at $M_{\text {miss }} \sim 1070 \mathrm{MeV} / c^{2}$. The green shaded area are events that have been identified as prompt Compton ones and are those left after subtraction of the other types of events. Even after this subtraction there is still some strength left at higher missing masses as seen in the green shaded region of figure $3 \mathrm{a}$. This strength is also believed to be due to $\pi^{0}$ events where a photon has been lost in the upstream hole of the CB but not yet properly accounted for. Analysis is still ongoing to try to better understand this.

Figure $3 \mathrm{~b}$ shows the events left after all background events have been subtracted with statistical errors shown. The red line at $M_{\text {miss }}=940 \mathrm{MeV} / \mathrm{c}^{2}$ shows the cut used when determining the experimental asymmetries. This cut was selected as it is believed that there might still be a significant contribution from $\pi^{0}$ events at higher $M_{\text {miss }}$ that are not yet properly accounted for. The events that survived this cut together with information on the beam and target polarisations were used to determine the experimental asymmetries with the method described in [13]. 


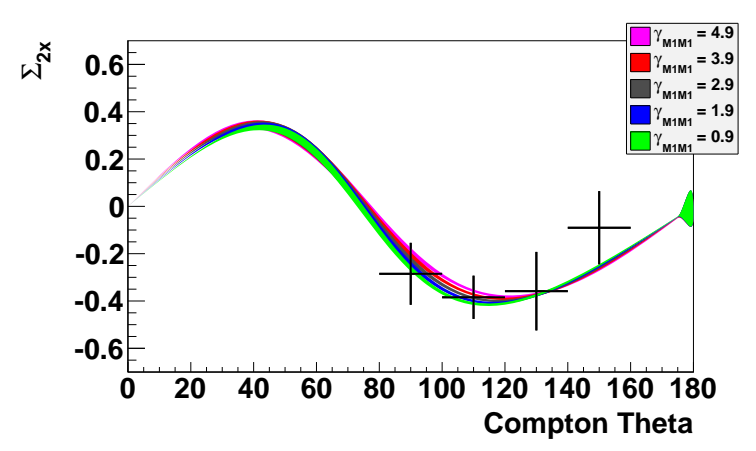

(a)

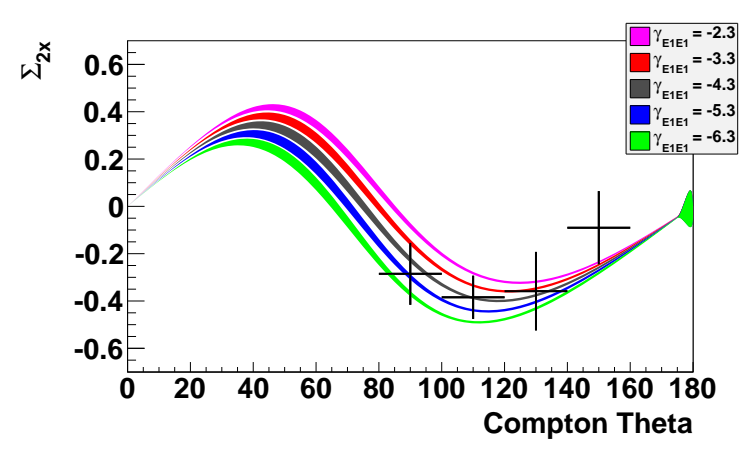

(b)

Figure 4: Experimental asymmetries for $273 \leq E_{\gamma} \leq 303 \mathrm{MeV}$ compared to different theoretical curves. In figure a the $\gamma_{M 1 M 1}$ contribution has been varied while the $\gamma_{E 1 E 1}$ was held fixed at -4.3 while for figure $\mathrm{b} \gamma_{E 1 E 1}$ was varied while $\gamma_{M 1 M 1}$ was held fixed at 2.9. See the text for more details.

\section{Preliminary Results}

The experimental $\Sigma_{2 x}$ asymmetry for $273 \leq E_{\gamma} \leq 303 \mathrm{MeV}$ is shown in figure 4 as a function of $\theta_{\gamma^{\prime}}$ compared to dispersion theory curves from [19]. The bands in each of the curves are from allowing the current values of $\alpha_{E 1}, \beta_{M 1}, \gamma_{0}$ and $\gamma_{\pi}$ to vary within their experimental errors. In figure 4a $\gamma_{E 1 E 1}$ was held fixed at -4.3 taken from [19] while $\gamma_{M 1 M 1}$ was varied. As can be seen from the figure the asymmetry is not strongly sensitive to the $\gamma_{M 1 M 1}$ variable and each of the values tested give a reasonable description of the experimental data. In figure $4 \mathrm{~b} \gamma_{M 1 M 1}$ was held fixed at 2.9 taken from [19] while $\gamma_{E 1 E 1}$ was varied. $\Sigma_{2 x}$ is more sensitive to $\gamma_{E 1 E 1}$ as seen in figure $4 \mathrm{~b}$ and it is possible to get a first experimental value for this quantity. A full $\chi^{2}$ fitting procedure is in development but as things stand with the analysis a $\gamma_{E 1 E 1}$ value of $-4.3 \pm 1.5 \times 10^{-4} \mathrm{fm}^{4}$ best describes the experimental asymmetries.

\section{Conclusions}

The double polarisation asymmetry, $\Sigma_{2 x}$, for the Compton scattering reaction off the proton has been measured for the first time for an incident photon energy range of $273 \leq E_{\gamma} \leq 303 \mathrm{MeV}$ and a scattered photon angular range of $80 \leq \theta_{\gamma^{\prime}} \leq 160^{\circ}$. This has allowed one of the four proton spin polarisabilities, $\gamma_{E 1 E 1}$, to be extracted individually for the first time. Comparison to dispersion relation calculations indicate that a value of $\gamma_{E 1 E 1}=-4.3 \pm 1.5 \times 10^{-4} \mathrm{fm}^{4}$ best describes the measured asymmetries.

\section{References}

[1] M.I. Levchuck and A.I. L'vov. Nucl. Phys. A, 674:449, 2000.

[2] M.-Th. Hütt, A.I. L'vov, A.I. Milstein, and M. Schumacher. Phy. Rep., 323:457, 2000.

[3] B. Pasquini, D. Drechsel, and M. Vanderhaeghen. Phys. Rev. C, 76:015203, 2007. 
[4] H.W. Grießhammer, J.A. McGovern, D.R. Phillips, and G. Feldman. Prog. Part. Nucl. Phys., 67:841, 2012.

[5] V. Olmos de León et al. Eur. Phys. J. A, 10:207, 2001.

[6] F. Federspiel et al. Phys. Rev. Lett., 67:1511, 1991.

[7] A. Zieger et al. Phys. Lett. B, 12:34, 1992.

[8] B.E. MacGibbon et al. Phys. Rev. C, 52:2097, 1995.

[9] J. Ahrens et al. Phys. Rev. Lett., 87:022003, 2001.

[10] H. Dutz et al. Phys. Rev. Lett., 91:192001, 2003.

[11] M. Camen. Phys. Rev. C, 65:032202, 2002.

[12] Martin Schumacher. Part. Nucl. Phys., 55:567, 2005.

[13] P.P. Martel. Measuring proton spin polarizabilities with polarized compton scattering. PhD thesis, University of Massachusetts Amherst, 2013.

[14] Thomas R. Hemmert, Barry R. Holstein, Joachim Kambor, and Germar Knöchlein. Phys. Rev. D, 57:5746, 1998.

[15] K.B. Vijaya Kumar, J.A. McGovern, and M.C. Birse. Phys. Lett. B, 479:167, 2000.

[16] George C. Gellas, Thomas R. Hemmert, and Ulf-G. Meißner. Phys. Rev. Lett., 85:14, 2000.

[17] D. Djukanovic. Virtual Compton Scattering in Baryon Chiral Perturbation Theory. PhD thesis, Johannes Gutenberg-Universitat Mainz, Germany, June 2008.

[18] D. Babusci, G. Giordano, A. I. L'vov, G. Matone, and A. M. Nathan. Phys. Rev. C, 58:1013, 1998.

[19] Barry R. Holstein, D. Drechsel, B. Pasquini, and M. Vanderhaeghen. Phys. Rev. C, 61:034316, 2000.

[20] S. Kondratyuk and O. Scholten. Phys. Rev. C, 64:024005, 2001.

[21] D. Drechsel, B. Pasquini, and M. Vanderhaeghen. Phys. Rep., 378:99, 2003.

[22] K.H. Kaiser et al. Nucl. Instr. and Meth. in Phys. Res. A, 593:159, 2008.

[23] J.C. McGeorge et al. Eur. Phys. J. A, 37:129, 2008.

[24] I. Anthony et al. Nucl. Inst. and Meth. A, 301:230, 1991.

[25] S.J. Hall et al. Nucl. Inst. and Meth. A, 368:698, 1996.

[26] K-H. Steffens, H.G. Andresen, J. Blume-Werry, and F. Klein. Nucl. Instr. and Meth. in Phys. Res. A, 325:378, 1993.

[27] A. Starostin et al. Phys. Rev. C, 64:055205, 2001.

[28] R. Novotny. The BaF-2 photon spectrometer TAPS. IEEE Trans. Nucl. Sci., 38:379, 1991.

[29] D. Watts. In Calorimetry in Particle Physics, Proceedings of the 11th International Conference, Perugia, Italy 2004. Edited by C. Cecchi, P. Cenci, P. Lubrano, and M. Pepe (World Scientific, Singapore, 2005, p. 560), 2005.

[30] G. Audit et al. Nucl. Inst. and Meth. A, 301:473, 1991.

[31] A. Thomas. Eur. Phys. J. Special Topics, 198:171, 2011. 